\title{
AVALIAÇÃO PRELIMINAR DO POTENCIAL DA PIRITA PARA GERAÇÃO DE ENERGIA
}

\author{
K. G. SANTOS ${ }^{1}$, C. M. OLIVEIRA ${ }^{2}$, N. C. FELTRIN ${ }^{3}$, L. O. FRAGA ${ }^{3}$ J. M. M. SILVA $^{1}$ e \\ M. PETERSON ${ }^{1}$
}

${ }^{1}$ Universidade do Extremo Sul Catarinense, Departamento de Engenharia Química

${ }^{2}$ Universidade Federal de Santa Catarina, Programa de Pós-graduação em Ciência e Engenharia de Materiais

${ }^{3}$ Centro de Educação Profissional Dario Geraldo Salles - Curso Técnico

E-mail para contato: kelvin_goularte@hotmail.com

\begin{abstract}
RESUMO - Dentre os rejeitos da extração e beneficiamento do carvão mineral, que demandam uma grande área física para descarte, encontra-se a pirita. Esse dissulfeto de ferro é o resíduo sólido de maior impacto ambiental, sendo responsável pela drenagem ácida de mina, gerada por seu contato com a água e/ou o oxigênio do ar. Além da hematita e do dióxido de enxofre, como resultado da oxidação da pirita há também liberação de energia. Este trabalho avalia a possibilidade de quantificação e aproveitamento da energia liberada na oxidação da pirita. O material de estudo, coletado no município de Treviso - SC, foi analisado por espectrometria de infravermelho por transformada de Fourier. O conteúdo energético da pirita foi determinado com base nos conceitos termodinâmicos. Sugeriu-se também um destino para os demais produtos da reação de oxidação.
\end{abstract}

\section{INTRODUÇÃO}

O carvão é uma das maiores fontes de energia não renovável do país e aproximadamente $60 \%$ de cada tonelada lavrada são resíduos sólidos (Nascimento, 2002). O principal componente desses resíduos é a pirita $\left(\mathrm{FeS}_{2}\right)$, que, apesar de relegada à condição de rejeito, pode ser empregada na produção de enxofre, ácido sulfúrico, hematita, dióxido de enxofre, fertilizantes (após formação de ácido sulfúrico) e sulfatos ferrosos (várias hidratações), produtos de maior valor agregado (Peterson, 2008).

Por apresentar elevado teor de impurezas, métodos de concentração são aplicados ao carvão mineral brasileiro para que ele atenda aos parâmetros exigidos pelas usinas termelétricas (Amaral Filho et al., 2013). A disposição dos resíduos sólidos (ricos em minerais sulfetados) gerados nesse processo é responsável pela formação de drenagens ácidas, disponibilizando metais bio-acumulativos que contaminam as águas e o solo (Evangelou, 1995).

Para conciliar extração do carvão mineral com desenvolvimento sustentável, é preciso dedicar atenção à pirita, tratando este mineral como matéria-prima para vários processos industriais (Peterson, 2008). 
Diante dos fatos expostos, o presente trabalho utiliza conceitos termodinâmicos para determinar a energia liberada na oxidação da pirita, bem como propõe alternativas de uso para a hematita e o dióxido de enxofre gerados na reação.

\section{PROCEDIMENTO EXPERIMENTAL}

O material de estudo foi coletado manualmente em uma pilha de rejeito de carvão mineral no município de Treviso (SC). A pirita foi britada em britador mandíbula, moída em moinho horizontal e, sequencialmente, moinho excêntrico com bolas de alta alumina. Verificou-se seu tamanho de partícula final em um granulômetro a laser Cilas 1064.

Para comprovar a presença de pirita e matéria orgânica no material, realizou-se um ensaio de espectrometria de infravermelho, a amostra foi preparada por prensagem no formato de pastilha contendo (aproximadamente) $95 \%$ de brometo de potássio $(\mathrm{KBr})$ e $5 \%$ de material a ser analisado. O equipamento utilizado foi um espectrofotômetro de FTIR marca Shimadzu, modelo IRPrestige-21 do IDT/UNESC. A análise foi realizada por transmitância, com uma velocidade de $0,2 \mathrm{~cm} / \mathrm{s}$ e uma resolução de $4 \mathrm{~cm}^{-1} \mathrm{com}$ intervalo de 400 a $4000 \mathrm{~cm}^{-1}$.

O poder calorífico superior do rejeito piritoso foi determinado conforme a norma LAB 510, utilizando um calorímetro adiabático. A quantidade de calor desprendida ao queimar completamente o material provocou um aquecimento no sistema e, consequentemente, uma diferença de temperatura $\Delta T$ na água contida no calorímetro. Com o $\Delta T$ e a capacidade calorífica (C) do calorímetro, empregou-se a equação 1 para calcular o poder calorífico superior (PC) pela combustão da massa $\mathrm{m}$.

$$
P C=\frac{C \Delta T}{m}
$$

Posteriormente, imergiu-se a pirita em água destilada a $60{ }^{\circ} \mathrm{C}, 0,02 \mathrm{~kg} / \mathrm{L}$, e o sistema foi submetido à agitação magnética (agitador Fisatom, modelo 752A). Na sequência, separou-se o mineral por filtração a vácuo (bomba a vácuo Prismatec, modelo 131) e a secagem do material ocorreu em estufa a vácuo Marconi, modelo MA 030/12 a $40{ }^{\circ} \mathrm{C}$.

A oxidação da pirita decorreu de um tratamento térmico em forno Jung tipo mufla (modelo tubular horizontal) em duas etapas: primeiro, aqueceu-se a amostra a $750{ }^{\circ} \mathrm{C} \mathrm{em}$ presença de oxigênio por uma hora; posteriormente, interrompeu-se a passagem de oxigênio e a temperatura foi elevada a $950{ }^{\circ} \mathrm{C}$ durante uma hora.

\section{RESULTADOS E DISCUSSÃO}

\subsection{Tamanho de partícula}

A Figura 1 traz a curva de distribuição de tamanho de partícula do rejeito piritoso após os processos de cominuição. 
Figura 1 - Curva de distridbuição de tamanho de partícula do rejeito piritoso.

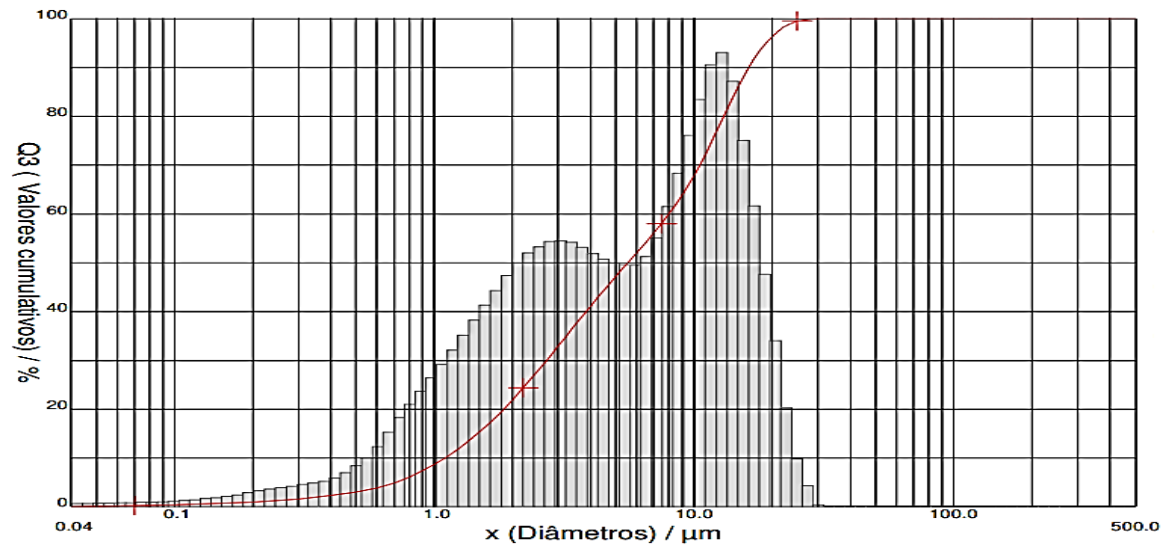

O diâmetro médio do material foi de $7,42 \mu \mathrm{m}$, Adequado para realizar a análise por difração de raios $\mathrm{X}$, além do tratamento térmico no forno Jung tipo mufla.

\subsection{Análise de FTIR}

Segundo Dunn et al. (1989a), em um espectro de FTIR, o pico característico da pirita é observado na região de $422 \mathrm{~cm}^{-1}$, representado na Figura 2 por um pico intenso em $414 \mathrm{~cm}^{-1}$

O espectro da Figura 2 também indica a presença de matéria orgânica no rejeito, pois o pico em $3236 \mathrm{~cm}^{-1}$ esta relacionado com ligações alifáticas, possivelmente oriundas do carvão mineral (Bandyopadhyay, 2005).

A banda forte e larga que começa em 3300 e é centrada em $3500 \mathrm{~cm}^{-1}$ é atribuída à umidade (Feng et al., 2015).

A amostra contém ainda carbonatos, picos em $1384 \mathrm{~cm}^{-1}$ e $1625 \mathrm{~cm}^{-1}$ (Evangelou, 1995), sulfatos de ferro, bandas no intervalo de 900 a $1200 \mathrm{~cm}^{-1}$ e a região entre 500 e 700 $\mathrm{cm}^{-1}$ (Dunn et al., 1992), e óxidos de ferro, região entre 472 e $530 \mathrm{~cm}^{-1}$ (Peterson, 2008).

Figura 2 - FTIR da pirita in natura

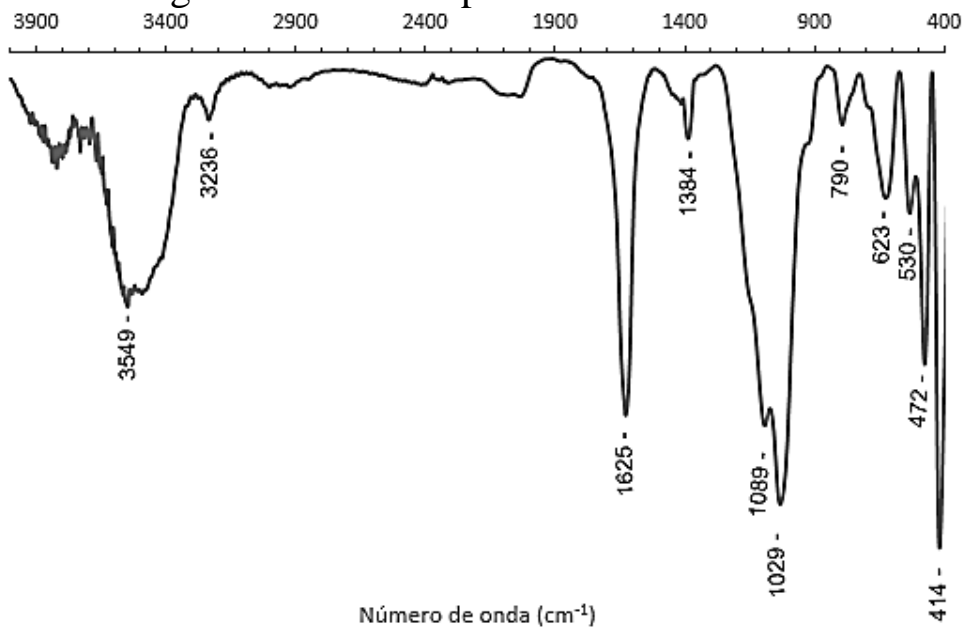




\subsection{Cálculo do calor de oxidação da pirita}

A oxidação da pirita pode ser representada pela Reação 2 .

$$
\mathrm{FeS}_{2(s)}+\frac{11}{4} \mathrm{O}_{2} \rightarrow \frac{1}{2} \mathrm{Fe}_{2} \mathrm{O}_{3(s)}+2 \mathrm{SO}_{2}(g)
$$

Utilizando a Lei de Hess (Equação 3) e conhecendo a entalpia padrão de formação das substâncias, é possível determinar o calor de reação.

Os dados estão dispostos na Tabela 1 .

Tabela 1 - Entalpia-padrão de formação (Perry et al., 1986).

\begin{tabular}{|c|c|}
\hline Substância & $\Delta \mathrm{H}_{\mathrm{f}}^{\circ}(\mathrm{Kcal} / \mathrm{mol})$ \\
\hline $\mathrm{FeS}_{2(\mathrm{~s})}$ & $-38,62$ \\
\hline $\mathrm{Fe}_{2} \mathrm{O}_{3}(\mathrm{~s})$ & $-198,5$ \\
\hline $\mathrm{SO}_{2}(\mathrm{~g})$ & $-70,94$ \\
\hline $\mathrm{O}_{2}(\mathrm{~g})$ & 0 \\
\hline
\end{tabular}

$$
\begin{aligned}
& \Delta H_{f}^{\circ}=\Sigma \Delta H_{f_{p}}^{\circ}-\Sigma \Delta H_{f_{r}}^{\circ}
\end{aligned}
$$

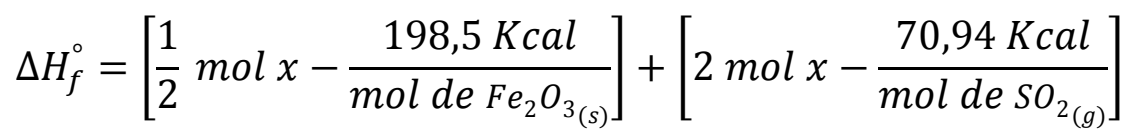

$$
\begin{aligned}
& -\left[1 \mathrm{~mol} x-\frac{38,62 \mathrm{Kcal}}{{\operatorname{mol~de~} \mathrm{FeS}_{2(s)}}_{2}}\right]-\left[\frac{11}{4} \operatorname{mol} x \frac{0 \mathrm{Kcal}}{\mathrm{mol} \mathrm{de} \mathrm{O}_{2(g)}}\right]
\end{aligned}
$$

$$
\Delta H_{f}^{\circ}=202,51 \mathrm{kcal}
$$

Desta forma, a oxidação de $1 \mathrm{~mol}$ de pirita promove a geração de 202,51 kcal de energia na forma de calor, equivalente a 1693,22 Kcal/Kg. Em termos mássicos, pode-se relacionar que a energia gerada por 3 toneladas de pirita oxidada equivale aquela produzida por 1 tonelada de carvão mineral brasileiro queimado (4000 Kcal/Kg).

\subsection{Cálculo do poder calorífico superior da pirita in natura}

Esse potencial energético resultou em $1738 \mathrm{Kcal} / \mathrm{Kg}$, comprovando a presença de carvão e matéria orgânica no rejeito, visto que o potencial calorífico foi superior ao calculado para a pirita in natura. 


\subsection{Rota de beneficiamento e aproveitamento energético da pirita} pirita.

A Figura 3 resume os processos propostos para o aproveitamento energético do mineral Figura 3 - Fluxograma do processo proposto.

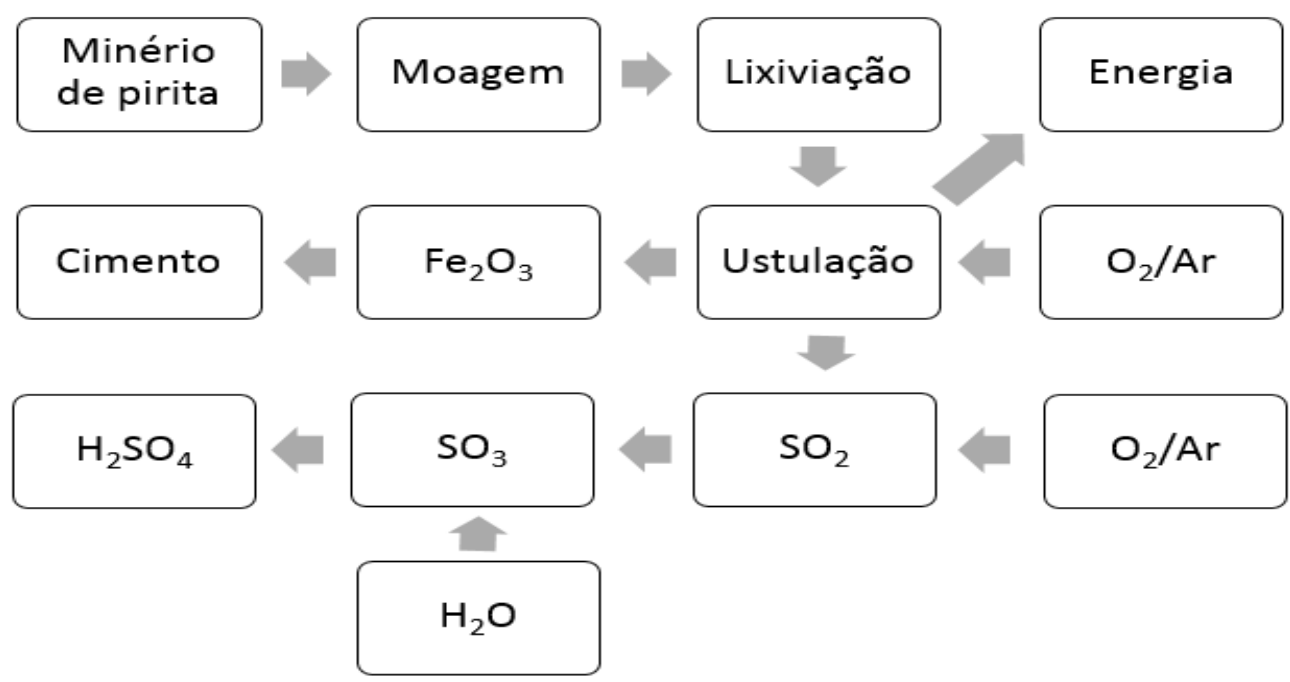

Em um primeiro momento, é proposta a geração de energia a partir do processo de ustulação da pirita (queima controlada).

A hematita gerada pode ser aplicada como aditivo para aumento da densidade do cimento. Outra utilização é na fabricação de sulfato ferroso, já estudada por Furmanski (2016).

O dióxido de enxofre é empregado na produção de ácido sulfúrico pelo processo de contato, onde o pentóxido de vanádio atua como catalisador durante a sua oxidação em trióxido de enxofre.

\section{CONCLUSÃO}

Pelos fatos apresentados, conclui-se que o conteúdo energético da pirita pode ser explorado. Porém, mais estudos devem ser feitos sobre o aproveitamento da hematita, para que todo o processo seja viável economicamente.

O ácido sulfúrico apresenta elevado valor comercial, podendo também ser utilizado dentro do processo para a fabricação de sulfato ferroso.

Para trabalhos futuros, aconselha-se realizar uma análise elementar do rejeito e identificar seu teor de carvão e pirita, comparando o valor energético real com o teórico.

\section{REFERENCIAS}


AMARAL FILHO, J. R. do; SCHNEIDER, I. A. H.; BRUM, I. A. S. de; SAMPAIO C. H.; MILTZAREK, G.; SCHNEIDER, C.. Caracterização de um depósito de rejeitos para o gerenciamento integrado dos resíduos de mineração na região carbonífera de Santa Catarina, Brasil. ver. Esc. Minas, v. 66, p. 347-353, 2013.

BANDYOPADHYAY, Debashis. Study of Kinetics of Iron Minerals in Coal by 57Fe Mössbauer and FT-IR Spectroscopy During Natural Burning. Hyperfine Interactions, v. 163, p. 167-176, 2005.

DUNN, J. G., DE, G. C., O'CONNOR, B. H. The effect of experimental variables on the mechanism of the oxidation of pyrite. Part 1. Oxidation of particles less than $45 \mu \mathrm{m}$ in size. Thermochim. Acta, v. 145, p. 115-130, 1989a.

DUNN, J. G., DE, G. C., O'CONNOR, B. H. The effect of experimental variables on the mechanism of the oxidation of pyrite. Part 2. Oxidation of particles of size 90-125 $\mu \mathrm{m}$. Thermochim. Acta, v. 155, p. 135-149, 1989b.

EVANGELOU, V. P. Pyrite oxidation and its control: solution chemistry, surface chemistry, acid mine drainage (AMD), molecular oxidation mechanisms, microbial role, kinetics, control, ameliorates and limitations, microencapsulations. Lexington: CRC Press, 1995.

FENG, Xiayu; CHEN, Wufeng; YAN, Lifeng. Free-standing dried foam films of graphene oxide for humidity sensing. Sensors and Actuators B: Chemical, v. 215, p. 316-322, ago. 2015.

FURMANSKI, L. M. Estudo da utilização de carepa de laminação de aço em processo de obtenção de sulfato ferroso. 115 p. Dissertação (Mestrado em Ciência e Engenharia de Materiais), Universidade do Extremo Sul Catarinense, Criciúma, 2016.

PERRY, R. H.; CHILTON, C. H. Manual de engenharia química. 5 ed. Rio de Janeiro: Guanabara Dois, 1986

PETERSON, M. Produção de sulfato ferroso a partir da pirita: desenvolvimento sustentável. 128 f. Tese (Doutorado em Engenharia Química), Universidade Federal de Santa Catarina, Florianópolis, 2008. 